\title{
Impact Of Environmental Conditions On Fingerprint Systems Performance
}

\author{
Abdarahmane WONE ${ }^{* \dagger}$, Joël DI MANNO*, Christophe CHARRIER ${ }^{\dagger}$ and Christophe ROSENBERGER ${ }^{\dagger}$ \\ *FIME EMEA, 14000 Caen, France \\ ${ }^{\dagger}$ Normandie Univ, UNICAEN, ENSICAEN, CNRS, GREYC, 14000 Caen, France \\ abdarahmane.wone@unicaen.fr, joel.dimanno@fime.com, christophe.charrier@unicaen.fr, christophe.rosenberger@ensicaen.fr
}

\begin{abstract}
Biometrics testing has for objective to determine the performance of a biometric system in order to guarantee security and user experience requirements. Providing trust in biometric systems is a key for many manufacturers. The performance is usually measured through the computation of matching scores between legitimate and impostor samples from a given database. Different bias in particular those linked to the environmental conditions can modify the performance of a biometric system. In this paper, we study the impact of acquisition conditions on fingerprint systems considering at the same time the quality and accuracy. We defined an own-made database controlling the acquisition conditions and we observe the behavior of three different matchers on these biometric data. Experimental results allow us to quantify their impact on performance and draw conclusions for testing biometric systems.
\end{abstract}

Index Terms-Real-life biometric testing, certification of fingerprint systems, acquisition conditions, environmental conditions, biometric performance.

\section{INTRODUCTION}

Biometrics is now a daily life technology to secure our devices for logical access control or payments. Experience has shown us that some interoperability issues appear when the product is used under non-expected environmental conditions. In some particular environments, the behavior of products can differ from what is calculated by testing laboratories. The standard certifications include performance testing by computing legitimate and impostor scores on a test database using pre-defined environmental parameters linked to final environments (temperature, light, humidity...) [1]. Final deployment of the product may be done in multiple different environmental conditions. This may introduce a bias which can have impact on interoperability by reducing security and user experience performance. For example, Payment systems using biometrics as Biometrics Sensor On Cards (BSOC) need to maintain high security and user experience in different parts of the world. Payment schemes have their own test plans for biometric products. Having a specific way to evaluate the impact of performance including variation of the environmental condition could help to increase trust on payment systems. Face and fingerprint based systems are mostly the firsts we might thought of. Indeed, we may observe some difficulties to be identified by a face recognition system when the light is not "good" (i.e., not close to experimental setup) or when there are some disturbing elements like non-desired source of light or people in the background. There are other uncontrolled factors such as the overexposure, tremors, misplaced finger during the acquisition, and so on. Some researchers investigated how to anticipate some of these unseen situations by training their algorithms on databases within particular situations [2, 3].

For fingerprint systems, the security and user experience is also affected when environmental conditions like temperature and humidity change. Annex C of ISO/IEC 19795-1 [4] gives a list of parameters that can be significant when testing a biometric system. Among other factors, environment is designated to impact the performance of a biometric system. It has been proven that matching rates are affected when we change the verification environment [3].

One of the motivations of this work is to address the two following questions:

- How acquisition conditions influence the performance, the security, the user experience and finally the trust of fingerprint systems?

- Are all fingerprint matchers impacted in a similar way? The paper is organized as follows. Section II briefly describes the related works that studied the impact of acquisition conditions to fingerprint systems. In section III, we present the designed experimental methodology. Experimental results are provided and discussed in sections IV and V. We conclude and give some perspectives of this study in section VI.

\section{RELATED WORK}

In the literature, to the best of our knowledge, very few works have studied the performance of biometric systems through different climatic environments. We can cite a recent work for finger veins [5]. Tan et al. [3] have shown that biometric matchers can have different behaviors through different environments. The study is more focusing on the PAIs (Presentation Attack Instruments) and the way they are detected with different PAD (Presentation Attack Detection) algorithms. Grosz et al. [6] give a module-by-module certification of a biometric process taking in consideration the moisture of the skin. Krishnasamy et al. [7] made a very interesting work for the recognition of fingerprints in wet conditions by building a wet and wrinkled fingerprint database available on demand. The study concludes that the error increases when matching a wet finger against a dry one. As clearly underlined by Fernandez-Saavedra et al. [8], the existing certification schemes such as FIDO alliance[1] or Common Criteria [9] are more focusing on 
the methodology and protocol of testing the performance of a biometric application under lab conditions, and/or ask biometric technology developers to include information on environmental influence and ways of reducing them.

Even if they point out the impact of environmental factors, all these standards and test methods only recommend defining and reporting the conditions of the tests which are naturally the lab conditions. Fernandez et al. [8] propose a full test protocol including the use of a climatic chamber to control the test conditions.

We propose in this paper to study the impact of both humidity and temperature on the performance of fingerprint systems. The proposed process is detailed in the next section.

\section{PROPOSED METHOD}

Based on our observations, texture of fingerprints change accordingly to the acquisition conditions. Those changes may badly influence quality of images and matching scores. Thus, we decided to analyze such bias and the effects that the environmental conditions could have on the performance of fingerprint systems. In this paper, we investigate if this assumption can align with experimental observations. This study can help some certification schemes for reducing interoperability risks by including evaluation of the bias linked to the acquisition environment of biometric products. Despite the good matching performance that we can have inside a testing laboratory using target application conditions, the perception of the end-user and the security can differ if product is used under unusual environmental conditions. In order to verify our assumption, a database has been created to simulate the uncontrolled environment.

\section{A. Data collection}

The unavailability of a controlled environmental fingerprint dataset enhanced our desire to build our own for the purpose of these experiments by setting up a test protocol to build the database. This database was built with a process respecting data privacy and security: the purpose of the experiment was explicitly provided, agreement forms explaining privacy rights, storage conditions, integrity and confidentiality of the data has been signed with all participants.

In a controlled climatic chamber, we create different environmental conditions with specific values of temperature and humidity. We collected 990 fingerprint sample images from 17 unique participants from 22 years old to 55 with $65 \%$ of males and $35 \%$ of females. For comparison, each set of the Fingerprint Verification Competition (FVC)[10] is around 880 images. We collected in each environment, 3 fingerprint scans of thumb, index and middle of both hands using a capacitive sensor, the Digital Persona's EikonTouch 700. The details of the built database is given in Table I.

We collected the fingerprint samples following the steps bellow:
- Step 1: Place the fingerprint sensor in the climatic chamber close to the specific testing hole

- Step 2: Configure Climatic Chamber with the targeted temperature and humidity conditions

- Step 3: Wait until stabilization

- Step 4: Volunteer puts their hand in the climatic chamber through the side hole

- Step 5: Remaining space around the hand is obstructed

- Step 6: Wait until stabilization

- Step 7: Capture fingerprint sample

1) Repeat for the 3 fingers of that hand

2) Back to step 4 with the other hand

- Step 8: Change conditions and back to Step 2

TABLE I: Environments considered in this study.

\begin{tabular}{|l|l|l|l|l|}
\hline $\begin{array}{l}\text { Environ- } \\
\text { ment }\end{array}$ & $\begin{array}{l}\text { Temp } \\
\left({ }^{\circ} \mathrm{C}\right)\end{array}$ & $\begin{array}{l}\text { Humidity } \\
(\%)\end{array}$ & $\begin{array}{l}\text { Number } \\
\text { of } \\
\text { fingers }\end{array}$ & $\begin{array}{l}\text { Total } \\
\text { images }\end{array}$ \\
\hline$\# 1$ & 15 & 20 & 54 & 162 \\
\hline$\# 2$ & 15 & 50 & 42 & 126 \\
\hline$\# 3$ & 15 & 80 & 72 & 216 \\
\hline$\# 4$ & 25 & 20 & 42 & 126 \\
\hline$\# 5$ & 25 & 50 & 48 & 144 \\
\hline$\# 6$ & 25 & 80 & 72 & 216 \\
\hline
\end{tabular}

\section{B. Validation protocol}

First, we score the quality of the acquired biometric samples in each environment computing the NFIQ2 fingerprint quality assessment metric [11], in order to be compliant with the ISO/IEC 29794-4:2017 recommendation [12]. Second, we need to consider the performance of a fingerprint system for each environment. We selected three fingerprint matching algorithms in order to generalize conclusions. We use the NIST (National Institute of Standards and Technology) matcher Bozorth 3 [13], the Minutia Cylinder-Code (MCC) matching algorithm [14, 15, 16, 17] and a commercial matcher. The commercial matcher uses its own minutiae extractor whereas for Bozorth 3, we use the MINDTCT [13] extractor. These extracted templates are converted to MCC templates.

In this work, we consider the authentication process for the performance evaluation of the three fingerprint matchers for the different environments. We compute the AUC values (Area Under Curve) for each scenario and matcher. The AUC value can be viewed as a measure ranking which is very useful and is based on pairwise comparisons between classifications of two classes. In other words, the AUC value is equal to the probability that a classifier will rank a randomly chosen positive instance higher than a randomly chosen negative one. That way, AUC can be considered as a global criterion of the performance. The higher the AUC is, better is the performance. We also estimate the confidence intervals (95\% confidence) of AUC results since the used database is not large. It is computed after a bootstrapping of 1000 times to confine the AUC value in a confidence interval with $95 \%$ of certitude. We 
also computes the EER (Equal Error Rate) and the FMR100. The EER represents the percentage of errors when the system is set as the false match rate is equal to the false non-match rate while the FMR100 is the highest achievable value of FNMR when the FMR is lower than $1 \%$. The lower the EER and FMR100 are, better is the performance.

\section{EXPERIMENTAL RESULTS}

In this section, the statistical analysis of obtained results are provided in order to evaluate the performance of the trial biometric systems under different environments.

\section{A. Quality Assessment}

The NFIQ2 score is used to assess the quality of the collected fingerprint samples through the different environments we created. NFIQ2 is a tool defined by the NIST which scores the quality of a fingerprint image between 0 to $100 \%$ according to the international biometric sample quality standard ISO/IEC 29794-1:2016. The distribution of the NFIQ2 scores for each environment is provided in Figure 1 . We can observe that the quality scores vary from very low (values close to $0 \%$ ) to very high quality (values close to $100 \%$ ) and cover the whole quality range.

We can also observe that the better average scores are obtained when the environment is dry for the two trial temperatures $\left(15^{\circ} \mathrm{C}\right.$ and $\left.25^{\circ} \mathrm{C}\right)$. Usually, when the humidity rate increases (whatever the considered temperature), we observe that the quality scores decrease. Indeed, when increasing the humidity, the sensor struggles to capture the samples because of the high moisture of the finger skin. People tend to press harder their finger on the sensor, which favors the decrease of the quality of the acquired sample. These results show that humidity deteriorates the quality of fingerprint samples.

\section{B. Matching comparison}

Since in many testing scenarios and in real-life situations, we perform the enrollment only once, we compare the collected samples in the climatic chamber against the reference samples (captured under nominal conditions: $22^{\circ} \mathrm{C}$ and $50 \%$ of humidity). Two fingerprint samples have been used for the computation of matching scores. We particularly have been interested in similarity of the AUC values (as global metric of the biometric system), EER and FMR100. For each detected minutiae, we have its coordinates, orientation and quality.

Table II gives the AUC of the different matchers we used as well as EER values and FMR100 values. When matching is done with reference enrolment condition template, we observe a variation between environments with different intensity. The two first matchers (Commercial and Bozorth) have a variation $<5 \%$ AUC, while MCC has a variation $>15 \%$ AUC. Matchers do not react in the same way while under different environmental conditions.
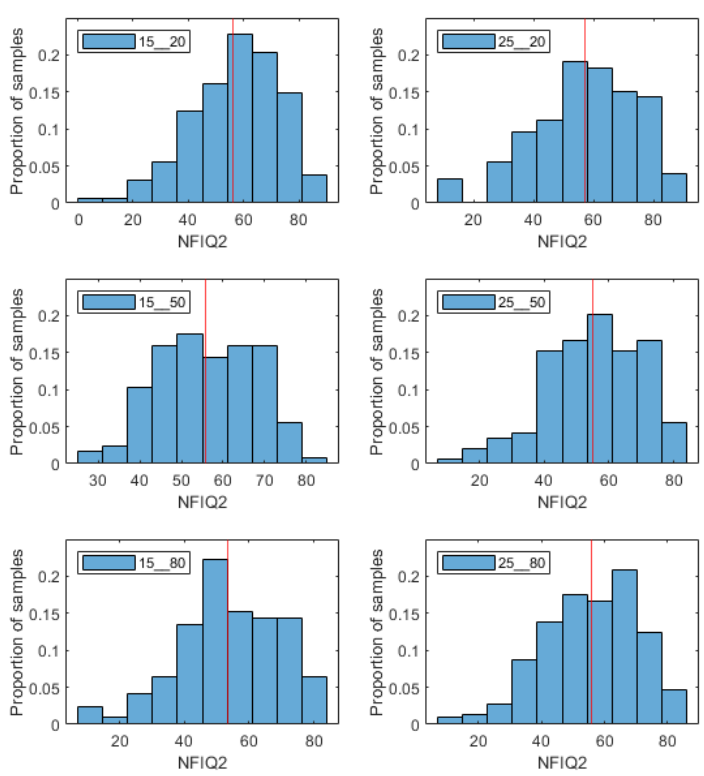

Fig. 1: Quality comparison of the whole database in different conditions: first column, Temperature $15^{\circ} \mathrm{C}$ and second column Temperature $25^{\circ} \mathrm{C}$, Humidity from $20 \%$ to $80 \%$ on each column with the average NFIQ2 value in each environment (vertical red line).

The MCC matcher seems to be more affected by the difference between the enrollment and the verification conditions. Indeed, it gives the lowest accuracy among the three matchers from the three accuracy metrics (AUC, EER and FMR100).

In addition, we wanted to investigate what would happen if the enrollment and the verification are performed within the same conditions. The goal is to evaluate how the performance less impacted by environmental conditions in this use case. We compute the performance of the trial matchers when the test subject performs the enrollment and the verification in the same environment. Even if for all matchers, AUC is increased when same conditions are used for enrollment and verification compared to when enrollment and verification are not done on the same environments, we notice that the impact of this increase depends of the matcher. The MCC matcher seems to be the most affected. For this matcher, when doing an authentication in the same environment that we registred, the global performance increases by $24 \%$ in average considering the AUC. The two other matchers are affected by around 1\%. Security and User Experience will be increased if enrolment can be redone in the targeted environment when the conditions change.

Table III gives the AUC value, EER and FMR100 of the different matchers when the enrollment is performed in the same conditions than the verification task.

To go further, and in order to analyze the influence each pa- 
TABLE II: Performance of the three matchers when enrollment is done in a "normal" environment (Temperature $22^{\circ} \mathrm{C}$, Humidity 50\%) and verification in different conditions: AUC in given with confidence interval (the higher is the AUC, better is the system).

\begin{tabular}{|c|c|c|c|c|c|c|c|c|c|}
\hline Env. & \multicolumn{3}{|c|}{ AUC (\%) } & \multicolumn{3}{c|}{ EER (\%) } & \multicolumn{3}{c|}{ FMR100 (\%) } \\
\hline & Commercial & Bozorth3 & MCC & Commercial & Bozorth3 & MCC & Commercial & Bozorth3 & MCC \\
\hline$\# 1$ & $97.83 \pm 0.04$ & $96.80 \pm 0.06$ & $74.54 \pm 0.19$ & 5.58 & 7.51 & 33.15 & 8.02 & 13.27 & 65.51 \\
\hline$\# 2$ & $97.39 \pm 0.04$ & $95.10 \pm 0.08$ & $65.10 \pm 0.20$ & 7.27 & 9.56 & 39.42 & 7.14 & 18.65 & 75.39 \\
\hline$\# 3$ & $99.08 \pm 0.03$ & $98.08 \pm 0.05$ & $68.78 \pm 0.21$ & 3.26 & 5.48 & 38.24 & 4.16 & 10.65 & 68.52 \\
\hline$\# 4$ & $99.45 \pm 0.02$ & $97.53 \pm 0.05$ & $80.33 \pm 0.16$ & 2.35 & 6.76 & 28.60 & 3.17 & 11.11 & 49.20 \\
\hline$\# 5$ & $94.75 \pm 0.06$ & $95.96 \pm 0.08$ & $77.14 \pm 0.18$ & 9.08 & 9.26 & 30.94 & 9.03 & 13.19 & 60.42 \\
\hline$\# 6$ & $97.83 \pm 0.05$ & $95.44 \pm 0.08$ & $69.07 \pm 0.22$ & 6.68 & 8.63 & 37.89 & 9.72 & 14.58 & 70.73 \\
\hline
\end{tabular}

TABLE III: Performance of the three matchers when enrollment and tests are done in the same environment: AUC in given with confidence interval (the higher is the AUC, better is the system).

\begin{tabular}{|c|c|c|c|c|c|c|c|c|c|}
\hline Env. & \multicolumn{3}{|c|}{ AUC(\%) } & \multicolumn{3}{c|}{ EER(\%) } & \multicolumn{3}{c|}{ FMR100(\%) } \\
\hline & Commercial & Bozorth3 & MCC & Commercial & Bozorth3 & MCC & Commercial & Bozorth3 & MCC \\
\hline$\# 1$ & $97.57 \pm 0.06$ & $94.55 \pm 0.10$ & $94.12 \pm 0.14$ & 7.37 & 10.94 & 8.90 & 9.26 & 14.81 & 25.92 \\
\hline$\# 2$ & $99.17 \pm 0.05$ & $97.53 \pm 0.09$ & $98.99 \pm 0.03$ & 1.19 & 3.89 & 6.39 & 11.91 & 10.71 & 14.28 \\
\hline$\# 3$ & $99.95 \pm 0.002$ & $99.08 \pm 0.02$ & $96.49 \pm 0.11$ & 1.39 & 5.46 & 6.70 & 13.89 & 7.64 & 15.27 \\
\hline$\# 4$ & $99.05 \pm 0.04$ & $98.89 \pm 0.03$ & $97.35 \pm 0.08$ & 3.62 & 6.14 & 9.29 & 3.57 & 10.71 & 16.66 \\
\hline$\# 5$ & $96.66 \pm 0.09$ & $97.70 \pm 0.05$ & $95.51 \pm 0.11$ & 7.16 & 7.86 & 10.50 & 11.46 & 11.46 & 18.75 \\
\hline$\# 6$ & $98.55 \pm 0.04$ & $97.12 \pm 0.06$ & $97.04 \pm 0.09$ & 4.10 & 7.81 & 7.59 & 4.86 & 13.89 & 20.93 \\
\hline
\end{tabular}

rameter can have on the global AUC value, we use a statistical hypothesis test to measure the dependency of acquisitions conditions with the performance of the fingerprint system. We compute the p-value of the AUC for each matcher. The p-values we obtain by observing one parameter at a time reveal that both humidity and temperature greatly influence the AUC of the commercial matcher. So, we assume the analyzed parameter is stationary and observe the p-value when only the other one is changing.

Tables IV and $\mathrm{V}$ give the different $\mathrm{p}$-values through all the possibilities. As illustration, for the 4th line of Table IV, we consider the situation when the humidity is static and equals to $50 \%$ and compute the p-value of the different AUC supposing it does not depend on the temperature. For Commercial and Bozorth 3 matchers, the p-value is $<5 \%$ meaning that the temperature has no significant impact. For MCC, the p-value is $>5 \%$ meaning that temperature has a significant impact on the AUC. This p-value method can highlight Security and User Experience risks for matcher when used under specific environmental conditions.

\section{DISCUSSION}

The work presented here deals with the problematic of fingerprints recognition from a matching point of view when the environmental conditions change. It brings an eye to a problematic that is not well underlined in the existing literature related to fingerpints.

Environmental factors can make biometrics challenging. This study shows how environmental bias impacts performance results, mainly by the temperature and humidity. We saw that some worldwide conditions can be reproduced on climatic chambers to obtain overview of security risk and user experience impact. We shown that the three trial matchers have their own vulnerability to specific conditions. Knowing this can prevent further challenges when products are deployed over the world and can help to provide more trust in biometric systems.

Due to the absence of available datasets, we had to build our database for the tests. We limited it to capacitive sensing as almost all the fingerprint sensors on cards are of this type. The test population is mainly composed of Caucasians at almost $90 \%$ which might make the results change when if the test is repeated. Nevertheless, this paper shows the variability of matching errors on genuine verification attempts through the environments and how it changes when the enrollment and the verification are done in the same conditions compared to doing the enrollment in a reference environment once and for all. To be fair, we will reinforce the test protocol to have a more representative world population by reconsidering the ethnicity and gender distribution of the test crew.

\section{CONCLUSIONS AND PERSPECTIVES}

In this paper, we present a preliminary work to understand the impact of acquisition conditions for the recognition of fingerprints. Experimental results highlighted there was a dependence between the performance of the matching algorithm and the environmental conditions. The trends of impacts of environmental conditions are shared between matchers but the strength of the bias is different. The p-values is usefull to characterize more precisely the factors linked to high bias impacts.

The MCC gives the worst performance when the tests samples are not from the same environment than the enrollment samples whereas it gives the best results on average when we perform a verification in the same conditions that we enrolled the test subject.

Future works will include the building of a larger database with different scanning technologies and recognition of the acquisition conditions considering a more balanced ethnicity and gender representation. This aims to find a future simulation 
TABLE IV: P-values considering separately the two parameters when enrollment and verification are done in the different conditions where $H_{T} \in[20,50,80]$ with $T_{E}$ and $H_{E}$ temperature and humidity at the enrollment and $T_{T}$ and $H_{T}$ temperature and humidity during the test (verification)

\begin{tabular}{||c|c|c|c||}
\hline Parameters & Commercial & Bozorth 3 & MCC \\
\hline$T_{E}=22^{\circ} C, T_{T}=15^{\circ} \mathrm{C}, H_{E}=50$ and $H_{T} \in\{20,50,80\}$ & $2.66 \times 10^{-5}$ & $7.99 \times 10^{-5}$ & $1.6 \times 10^{-3}$ \\
\hline$T_{E}=22^{\circ} \mathrm{C}, T_{T}=25^{\circ} \mathrm{C}, H_{E}=50$ and $H_{T} \in\{20,50,80\}$ & $2.01 \times 10^{-4}$ & $4.29 \times 10^{-5}$ & $2 \times 10^{-3}$ \\
\hline$H_{E}=50 \%, H_{T}=20 \%$ and $T_{T} \in\{15,25\}^{\circ} \mathrm{C}$ & $5.2 \times 10^{-3}$ & $2.4 \times 10^{-3}$ & $2.38 \times 10^{-2}$ \\
\hline$H_{E}=H_{T}=50 \%$ and $T_{T} \in\{15,25\}^{\circ} \mathrm{C}$ & $6.7 \times 10^{-3}$ & $2.9 \times 10^{-3}$ & $5.38 \times 10^{-2}$ \\
\hline$H_{E}=50 \%, H_{T}=80 \%$ and $T_{T} \in\{15,25\}^{\circ} \mathrm{C}$ & $4 \times 10^{-3}$ & $8.7 \times 10^{-3}$ & $1.4 \times 10^{-3}$ \\
\hline
\end{tabular}

TABLE V: P-values considering separately the two parameters when enrollment and verification are done in the same condition with $T_{E}$ and $H_{E}$ temperature and humidity at the enrollment and $T_{T}$ and $H_{T}$ temperature and humidity during the test (verification)

\begin{tabular}{||c|c|c|c||}
\hline Parameters & commercial matcher & Bozorth 3 & MCC \\
\hline$T_{E}=T_{T}=15^{\circ} C$, and $\left(H_{E}, H_{T}\right) \in\{20,50,80\} \times\{20,50,80\}$ & $4.98 \times 10^{-5}$ & $1.88 \times 10^{-4}$ & $1.65 \times 10^{-4}$ \\
\hline$T_{E}=T_{T}=25^{\circ} C$, and $\left(H_{E}, H_{T}\right) \in\{20,50,80\} \times\{20,50,80\}$ & $5.51 \times 10^{-5}$ & $2.84 \times 10^{-5}$ & $3.44 \times 10^{-5}$ \\
\hline$H_{E}=H_{T}=20 \%$ and $\left(T_{E}, T_{T}\right) \in\{20,50,80\} \times\{20,50,80\}$ & $4.8 \times 10^{-3}$ & $1.43 \times 10^{-2}$ & $8.7 \times 10^{-3}$ \\
\hline$H_{E}=H_{T}=50 \%$ and $\left(T_{E}, T_{T}\right) \in\{20,50,80\} \times\{20,50,80\}$ & $8.2 \times 10^{-3}$ & $4.49 \times 10^{-4}$ & $1.14 \times 10^{-2}$ \\
\hline$H_{E}=H_{T}=80 \%$ and $\left(T_{E}, T_{T}\right) \in\{20,50,80\} \times\{20,50,80\}$ & $6.4 \times 10^{-3}$ & $4.5 \times 10^{-3}$ & $1.8 \times 10^{-3}$ \\
\hline
\end{tabular}

solution to highlight environmental bias for Security Risk and User Experience without having to create physically different test environments.

\section{ACKNOWLEDGEMENTS}

This work is supported by Fime SAS and the French National Association for Research and Technology (ANRT). This work wouldn't be possible without the participation of Fime EMEA personnel who kindly accept to participate and give their fingers under different acquisition conditions.

\section{REFERENCES}

[1] S. Schuckers, G. Cannon, E. Tabassi, M. Karlsson, and E. Newton, "Fido biometrics requirements," Evaluation methodology, 2020. [Online]. Available: https://fidoalliance.org/specs/biometric/requirements/

[2] Z. Boulkenafet, J. Komulainen, L. Li, X. Feng, and A. Hadid, "Oulu-npu: A mobile face presentation attack database with real-world variations," 062017.

[3] B. Tan, A. Lewicke, D. Yambay, and S. Schuckers, "The effect of environmental conditions and novel spoofing methods on fingerprint anti-spoofing algorithms," in 2010 IEEE International Workshop on Information Forensics and Security, 2010, pp. 1-6.

[4] ISO, "Information technology - Biometric performance testing and reporting - part 1: Principles and framework," International Organization for Standardization, Geneva, CH, Standard ISO/IEC 19795-1:2006, 2006.

[5] S. Kirchgasser, C. Kauba, and A. Uhl, Towards Understanding Acquisition Conditions Influencing Finger Vein Recognition. Springer, Cham, 2020.

[6] S. Grosz, J. Engelsma, and A. Jain, "White-box evaluation of fingerprint recognition systems," 072020.

[7] P. Krishnasamy, S. Belongie, and D. Kriegman, in International Joint Conference on Biometrics (IJCB), Washington, DC, October 2011.
[8] B. Fernandez-Saavedra, R. Sanchez-Reillo, R. Moreno, and O. Miguel-Hurtado, "Environmental testing methodology in biometrics," 082010.

[9] C. Criteria, "Common criteria protection profile biometric verification mechanisms," Evaluation methodology, 2005.

[10] D. Maio, D. Maltoni, R. Cappelli, J. Wayman, and A. Jain, "Fvc2000: fingerprint verification competition," IEEE Transactions on Pattern Analysis and Machine Intelligence, vol. 24, no. 3, pp. 402-412, 2002.

[11] NIST, "Nfiq 2.0 nist fingerprint image quality," 2016.

[12] ISO Central Secretary, "Information technology — biometric sample quality - part 4: Finger image data," Geneva, CH, Standard ISO/IEC 29794-4:2017, 2017.

[13] W. J. S. Ko Kenneth, "User's guide to nist biometric image software (nbis)," Tech. Rep., 2007.

[14] R. Cappelli, M. Ferrara, and D. Maltoni, "Minutia cylinder-code: A new representation and matching technique for fingerprint recognition," IEEE transactions on pattern analysis and machine intelligence, vol. 32, pp. 2128-41, 122010.

[15] M. Ferrara, D. Maltoni, and R. Cappelli, "Noninvertible minutia cylinder-code representation," IEEE Transactions on Information Forensics and Security, vol. 7, pp. 1727-1737, 122012.

[16] R. Cappelli, M. Ferrara, and D. Maltoni, "Fingerprint indexing based on minutia cylinder-code," IEEE transactions on pattern analysis and machine intelligence, vol. 33, pp. 1051-7, 122010.

[17] M. Ferrara, D. Maltoni, and R. Cappelli, "A two-factor protection scheme for mcc fingerprint templates," Lecture Notes in Informatics (LNI), Proceedings - Series of the Gesellschaft fur Informatik (GI), pp. 171-178, 012014. 